\title{
Inflammatory Infiltrates and Neovessels Are Relevant Sources of MMPs in Abdominal Aortic Aneurysm Wall
}

\author{
Christian Reeps $^{\mathrm{a}} \quad$ Jaroslav Pelisek $^{\mathrm{a}}$ Stefan Seidl $^{\mathrm{b}}$ Tibor Schuster $^{\mathrm{c}}$ \\ Alexander Zimmermann $^{a}$ Andreas Kuehnla Hans-Henning Eckstein ${ }^{a}$ \\ ${ }^{a}$ Department of Vascular Surgery, ${ }^{\mathrm{b}}$ Institute of Pathology and ${ }^{\mathrm{C} I n s t i t u t e}$ of Epidemiology and Medical Statistics (IMSE), \\ Klinikum rechts der Isar der Technischen Universität München, Munich, Germany
}

\section{Key Words}

Aneurysm • Matrix metalloproteinases • Inflammation •

Neovascularization

\begin{abstract}
Objectives: Abdominal aortic aneurysm (AAA) wall is characterized by degradation of extracellular matrix through matrix metalloproteinases (MMPs), chronic inflammatory cell infiltration and extensive neovascularization. So far, MMP expression within AAA wall in association with infiltrates and neovascularization has not yet been studied. Methods: Vessel walls of 15 AAA patients and 8 organ donors were analyzed by immunohistochemistry for expression of various MMPs (MMP-1, $-2,-3,-7,-8,-9,-12$ and -13) in all cells located within the AAAs and correlated with infiltrates and neovascularization. Results: Luminal endothelial cells (ECs) were positive for MMP-1, -3 and -9 , ECs of mature neovessels were furthermore positive for MMP-2. Immature neovessels expressed all MMPs tested except for MMP-13. Aortic medial smooth muscle cells (SMCs) expressed MMP-1, $-2,-3$ and -9 , SMCs of mature neovessels, only MMP-1, -3 and -9. Inflammatory infiltrates expressed all MMPs tested except for MMP-2, macrophages expressed all MMPs. Infiltrates
\end{abstract}

\section{KARGER}

Fax +41613061234 E-Mail karger@karger.ch www.karger.com
(C) 2009 S. Karger AG, Basel

$1015-2008 / 09 / 0765-0243 \$ 26.00 / 0$

Accessible online at:

www.karger.com/pat were composed mainly of B cells (58.5 $\pm 10.9 \%)$ and T lymphocytes $(26.3 \pm 9.5 \%)$. Furthermore, significant inverse correlations were found between the amounts of inflammatory cells, neovessels and collagen/elastin content of the aortic vessel wall $(r=+0.806 / p<0.001, r=-0.650 / p=0.012$, $r=-0.63 / p<0.015$; respectively). Conclusion: Inflammatory infiltrates and invading neovessels are relevant sources of MMPs in the AAA wall and may substantially contribute to aneurysm wall instability.

Copyright $\odot 2009$ S. Karger AG, Basel

\section{Introduction}

Despite considerable advances in surgical treatment, the prognosis of ruptured abdominal aortic aneurysms (AAAs) is still poor with a $75-85 \%$ mortality rate [1-3]. Expression of matrix metalloproteinases (MMPs) is commonly associated with degradation of aortic vessel wall but the source of MMPs has not yet been completely elucidated [4-7]. Furthermore, AAA formation and rupture

The first two authors have equally contributed to this work. 


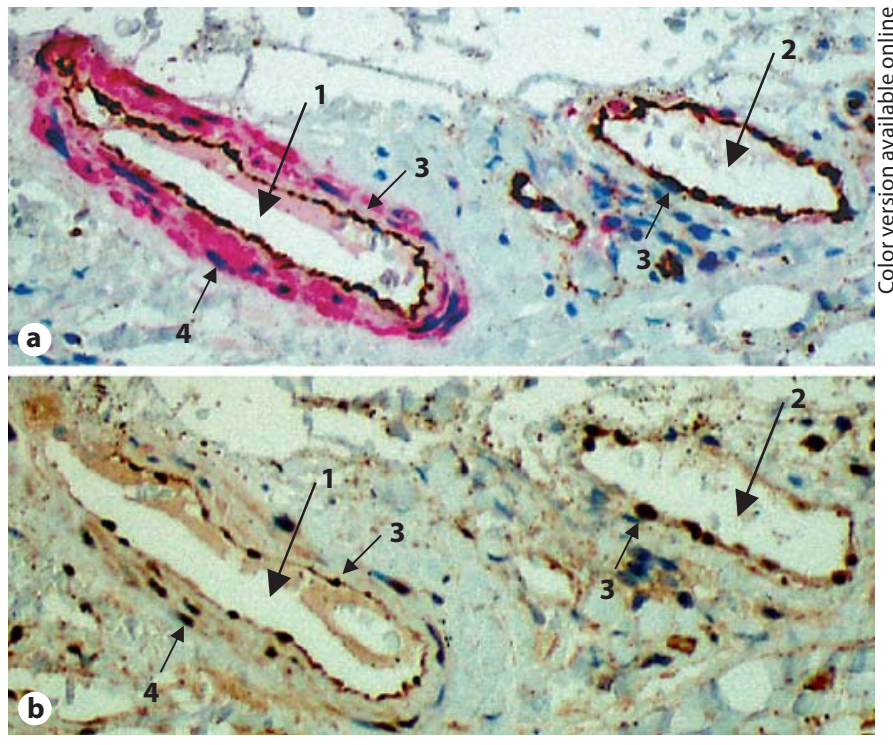

Fig. 1. Example of consecutive staining to assess MMP expression on individual cells of an AAA vessel wall. Consecutive slides were stained with an appropriate $\mathrm{Ab}$ against various cell types in the aortic vessel wall and MMPs of interest. a CD31/SMA (ECs/SMCs) double staining of neovessels. $\mathbf{b}$ The following slide was stained for MMP-1. 1 = Mature neovessel; 2 = immature neovessel; 3 = ECs; 4 = SMCs.

are closely accompanied by inflammation and neovascularization $[8,9]$. MMP-2 and -9 are so far the most intensively investigated MMPs in human AAAs [10-13]. Moreover, considerably enhanced expression of MMP- 8 and -9 was observed at AAA rupture sites [14-16] and upregulation of MMP-1, -7, -9 and -12 was observed in diseased aneurysm walls [16]. In addition to extracellular matrix degradation, extensive neovascularization $[8,9,17-19]$ with surrounding accumulation of inflammatory cells [6, $9]$ is frequently observed. However, there is some variation in the description of the cellular components of the infiltrates. While some studies detected up to $90 \%$ of the inflammatory cells as T helper cells [20-22], others describe $B$ lymphocytes as the major cell component in AAAs $[23,24]$.

Interestingly, MMP expression within the aortic aneurysmal vessel wall in association with neovascularization and inflammatory infiltrates has not been investigated yet. The aim of the current study was thus to characterize inflammatory cells and to compare MMP expression within AAAs in correlation with neovessels and infiltrates. Selected members of all soluble MMP subgroups, i.e., collagenases (MMP-1, -8, -13), gelatinases
(MMP-2, -9), stromelysins/matrilysins (MMP-3, -7) and others (metalloelastase MMP-12) [2, 25], were analyzed and their expression was investigated in histological samples from AAA patients.

\section{Patients and Methods}

\section{Patients and Tissue Collection}

Samples of 15 patients (14 males, 1 female) with asymptomatic AAA were obtained during conventional surgical repair. All tissue samples were collected in a standardized manner from the anterior sac of the infrarenal abdominal aorta. Average age was $70.8 \pm 5.4$ (range 62-80 years) and average maximum AAA diameter size determined by CT scan was $5.5 \pm 0.9 \mathrm{~cm}$ (range $4.5-$ $7.2 \mathrm{~cm}$ ). Aortic tissue from 8 organ donors was used as a control (all males, average age 64 years). Subsequent tissue samples were placed in formalin, immediately embedded in paraffin and stored appropriately for further histological and immunohistochemical analyses. The study was conducted with the approval of our institutional review board and informed consent was obtained from all patients prior to their participation in the study. The investigation conforms to the principles outlined in the Declaration of Helsinki for use of human tissue or subjects [26].

\section{Immunohistochemistry}

Histological and immunohistochemical analyses were performed on representative sections $(2-3 \mu \mathrm{m})$ of aortic tissue samples. Paraffin sections were routinely stained with hematoxylineosin (HE) and elastin-van Gieson (EvG) to assess tissue morphology, cellular composition, degree of infiltration with inflammatory cells and the content of elastin and collagen fibers of all AAA samples. For immunohistochemistry, dewaxed and hydrated tissue section samples were boiled to retrieve antigen epitopes, washed and treated with appropriate antibodies (Abs).

For analysis of cells within the aneurysm wall, the following primary Abs were used: vascular smooth muscle cells (VSMCs, anti smooth muscle actin, SMA, clone HHF35, dilution 1:200; Dako, Hamburg, Germany), endothelial cells (CD31 anti-von Willebrand factor VIII, clone F8/86, dilution 1:500; Dako/CD31, clone JC70A, dilution 1:40; Dako; due to a higher sensitivity, factor VIII was used in this study), macrophages/monocytes (antiCD68, clone KP1, dilution 1:2,000; Dako), T lymphocytes (antiCD3, rabbit polyclonal, dilution 1:400; Dako), B lymphocytes (anti-CD20, clone L26, 1:500; Dako), natural killer cells (NK cells, anti-CD56, clone 1B6, dilution 1:100; Dako), plasma cells (clone VS38c, dilution 1:500; Dako). For detection of MMP expression, the following Abs were applied: MMP-1 (epitope-specific rabbit polyclonal Ab, dilution 1:100; LabVision, Wedel, Germany), MMP-2 (mouse monoclonal Ab, clone Ab-4, dilution 1:50; LabVision), MMP-3 (mouse monoclonal Ab, clone Ab-2, dilution 1:100; LabVision), MMP-7 (mouse monoclonal Ab, clone Ab-4, dilution 1:100; LabVision), MMP-8 (mouse monoclonal Ab, clone 100608, dilution 1:100; R\&D System, Wiesbaden, Germany), MMP-9 (epitope-specific polyclonal $\mathrm{Ab}$, clone $\mathrm{Ab}-4$, dilution 1:100; LabVision), MMP-12 (mouse monoclonal $\mathrm{Ab}$, clone $\mathrm{Ab}-4$, dilution 1:400; R\&D System), MMP-13 (mouse monoclonal Ab, clone Ab4, dilution 1:20; LabVision). 
Fig. 2. Representative pathophysiological composition of an aortic vessel wall of a patient with AAA (a) and a normal healthy aorta (b). Hemalaun-eosin staining was used to show the cellular content of the sample (blue); factor VIII staining demonstrates neovessels (red). ${ }^{*}=$ Atherosclerotic lesion in the intimal region; \# = media with considerably less SMCs compared to control aorta; $\ddagger=$ inflammatory infiltrates; arrow $=$ neovessels associated with inflammatory cells reflect markedly enhanced neovascularization within AAA compared to control aorta.
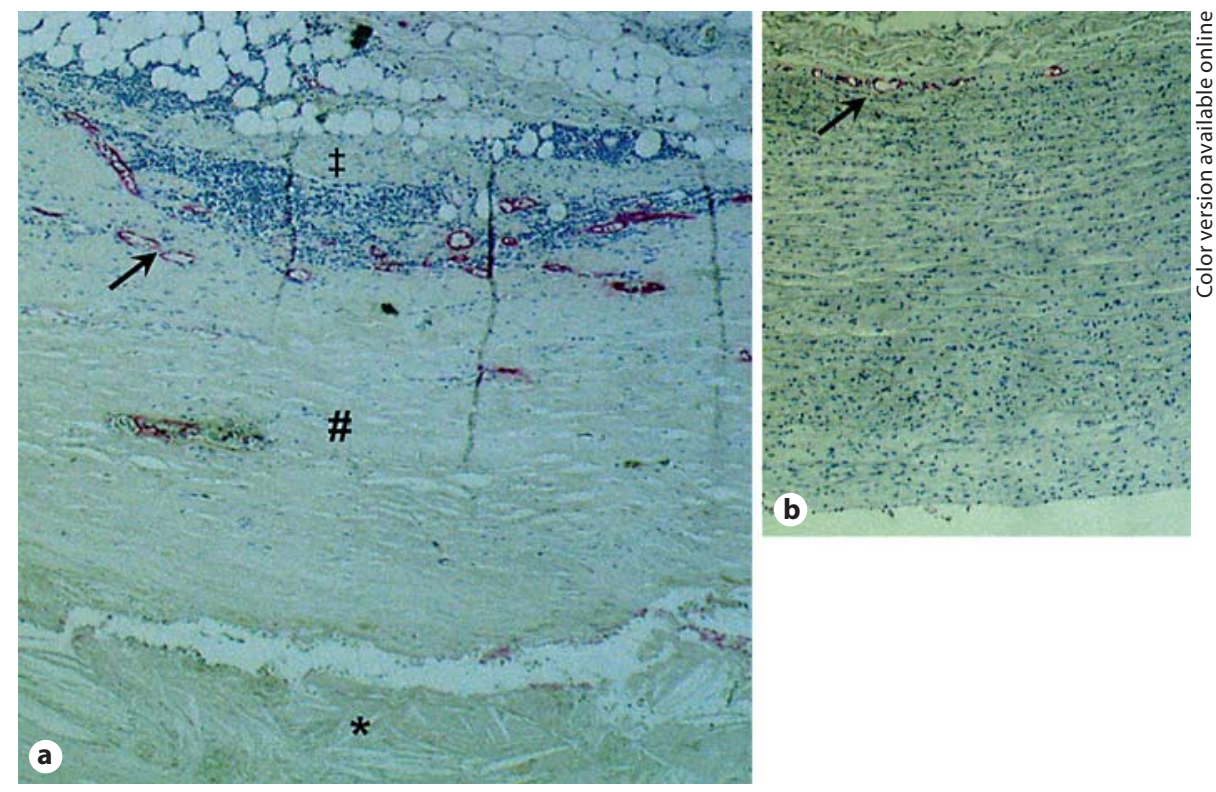

Following primary antibody incubation, factor VIII and SMA were visualized using the APAAP ChemMate Detection Kit (rabbit anti-mouse secondary Ab; Dako) according to the manufacturer's instructions. All other primary Abs were detected and visualized by peroxidase/DAB ChemMate Detection Kit (biotinylated goat anti-mouse/anti-rabbit secondary Ab; Dako).

For determining MMP expression in the individual cells within the AAA vessel wall, consecutive slides from each specimen were prepared and incubated with the appropriate antibodies. In all cases, one slide was stained with an Ab to detect the specific cell type (VIII/CD31, SMA, CD68, CD3, CD20, CD56, or VS38c) and a consecutive slide was stained with Ab against the MMP of interest (MMP-1, -2, -3, -7, -8, -9, -12 and -13). An example of the colocalization study is shown in figure 1 with CD31/SMA double staining of two different neovessels in an AAA and the consecutive slide stained with MMP-1.

\section{Histological Evaluation and Statistical Analysis}

The intensity of staining for MMPs was first evaluated in all specimens to validate the different staining intensities for each sample. Histological specimens were then graduated semiquantitatively as follows: (-) no staining; (+) positive staining of most cells and the majority of specimens; (++) strong overall positive staining detected in all cells and all specimens; $(-/+)$ intensity of staining varied between different samples and also within the same specimen; $(-/++)$ heterogeneous staining results from $(-)$ to $(++)$.

The percentage of inflammatory cells was determined by counting cells positive for CD3, CD20, CD68, CD56, or VS38c and compared with the total cell number of the corresponding inflammatory area by 2 observers blinded to the source of each specimen. Data were analyzed using the Friedman test for comparisons of related samples followed by multiple post hoc comparisons. Data correlations were performed by calculating the Spearman rank correlation coefficient. Values are shown as mean \pm SD. $p<0.05$ was considered as significant.

MMPs, Inflammation and

Neovascularization in AAA

\section{Results}

\section{Structural Composition of AAAs}

Typical examples of the vessel wall of an advanced human AAA ( $7.3 \mathrm{~cm}$ max. diameter) and healthy aortic tissue are shown in figure 2. Aneurysmal tissue contained a typical atherosclerotic plaque in the tunica intima with surrounding macrophages and foam cells. In addition, enhanced neovascularization was observed mainly in the tunica media and partially in the intimal layer. Neovessels were predominantly associated with inflammatory infiltrates. Furthermore, the majority of these new microvessels were immature with an EC layer without surrounding pericytes or SMCs. In control aortic tissue, microvessels were found only at the border between the media and adventitia and within the adventitia.

\section{MMP Expression in ECs and SMCs in Neovessels within AAAs}

First, MMP expression was analyzed in ECs and SMCs of neovessels within the aneurysmal lesions (table 1). In addition, to prove whether differences in MMP activities depend on the character of the new vessels, immature neovessels only containing an endothelial layer were compared with mature microvessels with surrounding SMCs. Regarding MMP-1, -2, -3 and -9, strong expression was found in ECs of both immature and mature neovessels (fig. $3 \mathrm{a}-\mathrm{c}, \mathrm{f}$ ). In addition, mature neovessels expressed MMP-1 and -3 in the adjacent SMC layer 
Fig. 3. Immunohistochemical staining of MMP-1 (a), MMP-2 (b), MMP-3 (c), MMP7 (d), MMP-8 (e), MMP-9 (f), MMP-12 (g) and MMP-13 (h) in neovessels. Two different types of neovessels are shown, i.e., immature neovessels in the upper part of each example and mature neovessels comprising the endothelial layer and surrounding pericytes/SMCs in the lower part. Arrows show ECs $\left(^{*}\right)$ and SMCs (\#).
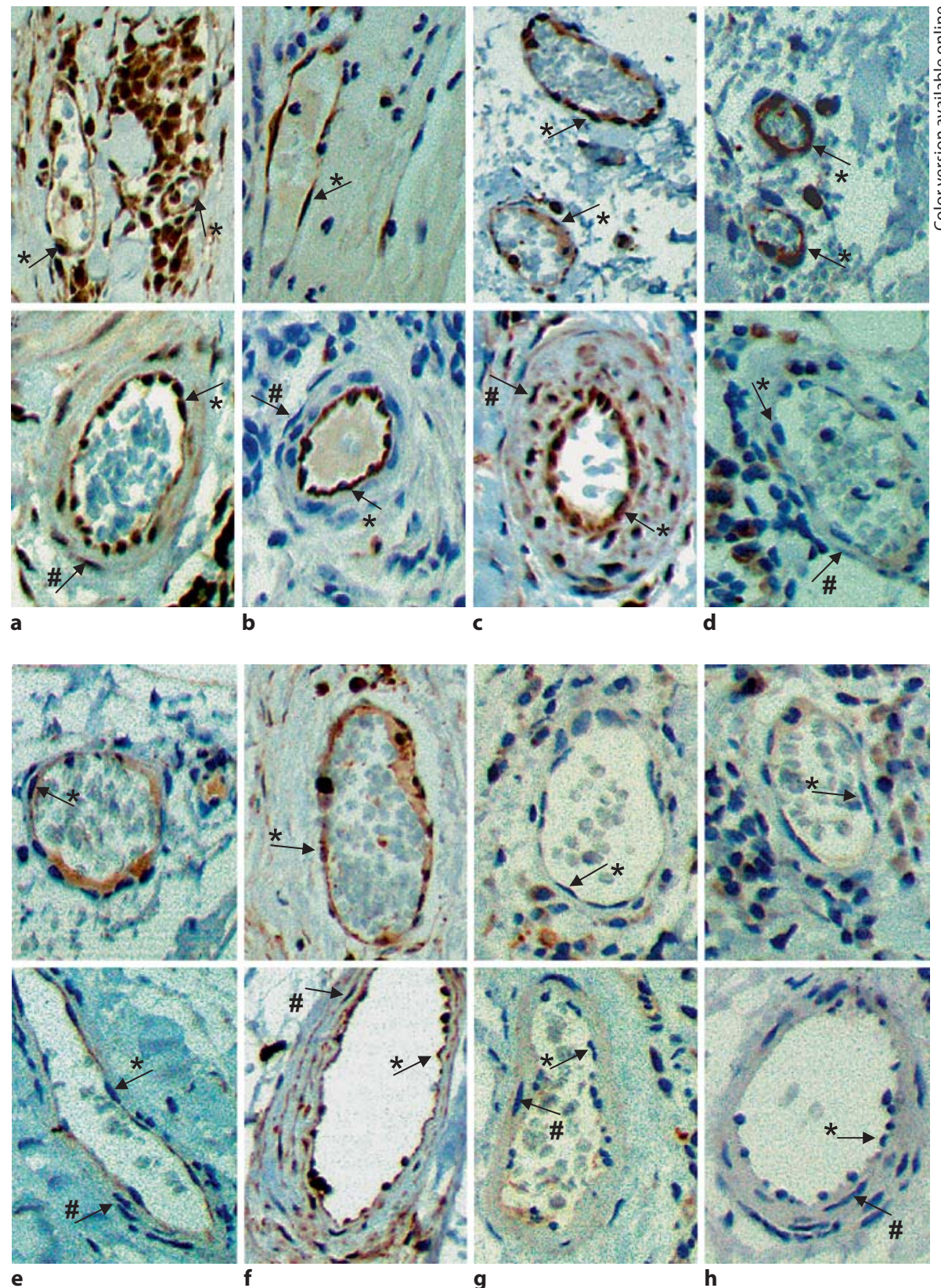

h

(fig. 3a, c, lower part). MMP-7, -8 and -12 expressions were found only in small immature neovessels, not in mature neovessels (fig. 3d, e, g). No MMP-13 expression was detected in neovessels within the aneurysmal vessel wall (fig. 3h).

\section{MMP Expression in Luminal ECs and Medial}

SMCs within AAAs

MMP-1 and -9 were expressed in luminal ECs of thrombus-free luminal aortic wall (fig. $4 \mathrm{a}, \mathrm{f}$, table 1).
From the remaining MMP subgroups, only MMP-3 was partially expressed in these cells (fig. 4d). The expression of MMP-1 and -9 in medial SMCs corresponded to their expression in luminal ECs (fig. 4a, f). In contrast to luminal ECs, all medial SMCs were positive for MMP-3 (fig. 4c). Furthermore, MMP-2 expression was observed in medial SMCs (fig. 4b) but not in SMCs surrounding ECs of mature neovessels (fig. 3b). 


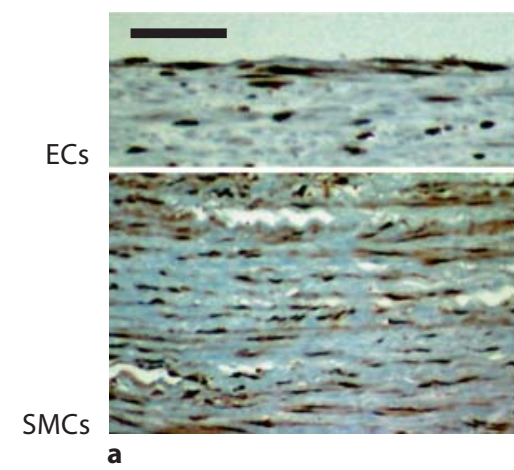

a

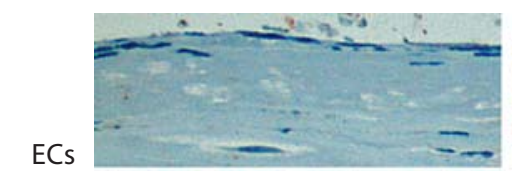

SMCs

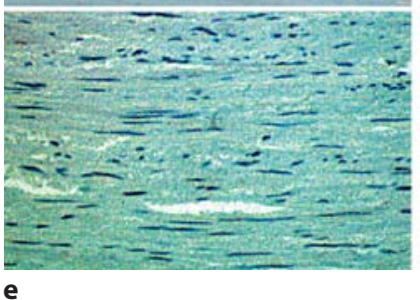

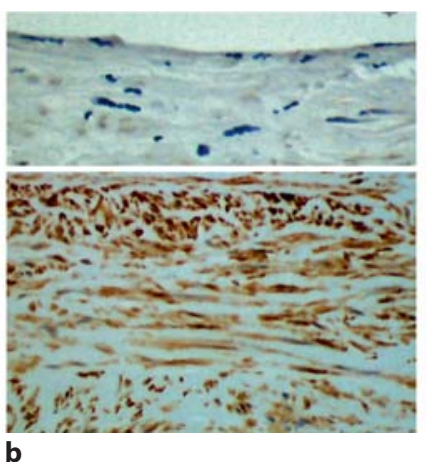
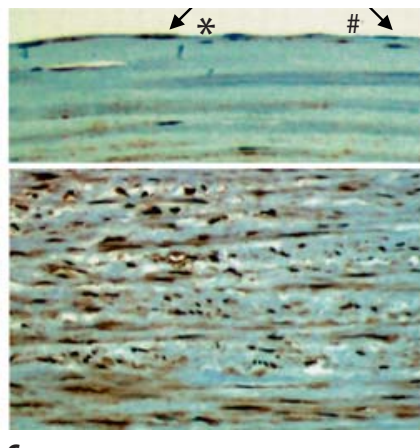

c

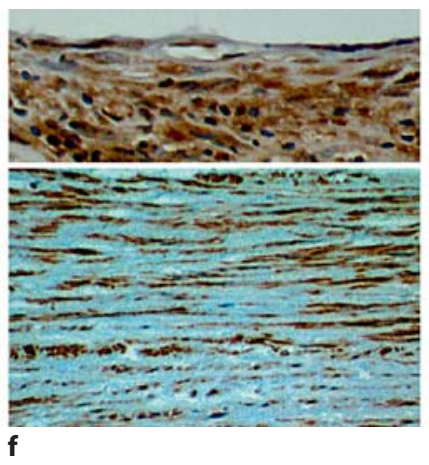

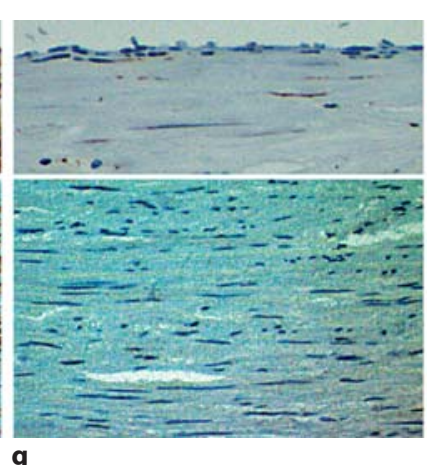

g
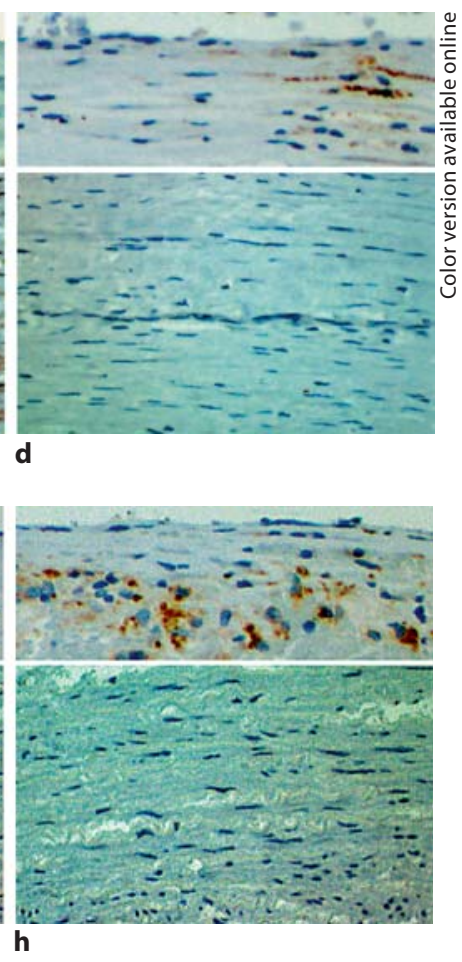

Fig. 4. Immunohistochemical staining of MMP-1 (a), MMP-2 (b), MMP-3 (c), MMP-7 (d), MMP-8 (e), MMP-9 (f), MMP-12 (g) and MMP-13 (h) in luminal ECs and medial SMCs of human AAA samples. The bar in a corresponds to $50 \mu \mathrm{m}$ and also applies to $\mathbf{b}-\mathbf{h}$. c Some endothelial cells were positive $\left({ }^{*}\right)$, others were negative (\#) for MMP-3.

Table 1. Semiquantitative analysis of MMP expression in different cells located within AAAs

\begin{tabular}{|c|c|c|c|c|c|c|c|}
\hline & \multirow{3}{*}{$\begin{array}{l}\text { Luminal } \\
\text { ECs }\end{array}$} & \multirow{3}{*}{$\begin{array}{l}\text { Medial } \\
\text { SMCs }\end{array}$} & \multirow{3}{*}{$\begin{array}{l}\text { Macro- } \\
\text { phages }\end{array}$} & \multirow[t]{3}{*}{ Infiltrates } & \multicolumn{3}{|l|}{ Neovessels } \\
\hline & & & & & \multirow{2}{*}{$\frac{\text { immature }}{\text { ECs }}$} & \multicolumn{2}{|c|}{ mature } \\
\hline & & & & & & ECs & SMCs \\
\hline MMP-1 & ++ & ++ & ++ & ++ & ++ & ++ & ++ \\
\hline MMP-2 & - & ++ & $+/++$ & - & $+/++$ & $+/++$ & - \\
\hline MMP-3 & $-1+$ & + & ++ & ++ & ++ & ++ & ++ \\
\hline MMP-7 & - & - & + & $-/++$ & $-/++$ & - & - \\
\hline MMP-8 & - & - & + & $-/++$ & $-/++$ & - & - \\
\hline MMP-9 & ++ & ++ & ++ & ++ & ++ & ++ & ++ \\
\hline MMP-12 & - & - & + & $-/+$ & $-/++$ & - & - \\
\hline MMP-13 & - & - & + & + & - & - & - \\
\hline
\end{tabular}

Semiquantitative evaluation was performed as follows: ' ++ ' $=$ strong positive staining of all cells and all specimens analyzed; ' + ' = positive staining of most cells and the majority of specimens; '-' = no staining; ' $-/+$ ' = not all cells staine positive, staining intensity varied; ' $-/++$ ' = heterogeneous staining between ' - ' and '十+'. 


\section{Composition of Inflammatory Cells within AAAs}

Because not all inflammatory cells were positive for MMPs, the individual cell type of inflammatory infiltrates within AAA walls were analyzed by immunohistochemistry and counting of the stained cells (table 2). The majority of these cells were identified as B lymphocytes with $58.5 \pm 10.9 \%$ of total inflammatory cells, followed by $\mathrm{T}$ lymphocytes with $26.3 \pm 9.5 \%$. However, B and T cell distribution was rather irregular (table 2, see corresponding ranges). Other inflammatory cells found in the infiltrates were plasma cells $(7.7 \pm 6.6 \%)$, NK cells (3.1 \pm $2.3 \%)$ and monocytes $(4.5 \pm 1.3 \%)$. The quantity of plasma cells varied between sporadic and up to $32 \%$ of the total inflammatory cells (table 2). The distribution of inflammatory cells within AAA walls showed significant differences between all cell types $(p<0.001)$. Multiple post hoc comparison between the individual inflammatory cells also showed significant disparity: T lymphocytes (CD3) versus NK cells, plasma cells, monocytes/ macrophages $(p<0.05)$, B cells $(C D 20)$ versus NK cells, plasma cells, monocytes/ macrophages $(\mathrm{p}<0.05)$ and NK cells $(\mathrm{CD} 56)$ versus plasma cells $(\mathrm{p}<0.05)$.

Table 2. Cellular composition of infiltrates within AAAs

\begin{tabular}{lcc}
\hline Cells & Mean \pm SE, \% & Range, \% \\
\hline B lymphocytes & $58.5 \pm 10.9$ & $18.1-83.7$ \\
T lymphocytes & $26.3 \pm 9.5$ & $10.5-68.1$ \\
Plasma cells & $7.7 \pm 6.6$ & $2.2-32.3$ \\
Monocytes & $4.5 \pm 1.3$ & $1.4-7.5$ \\
NK cells & $3.1 \pm 2.3$ & $0.5-6.9$ \\
\hline
\end{tabular}

Overall comparison between individual cell types: $\mathrm{p}<0.001$. Significant differences in post hoc pair comparisons $(\mathrm{p}<0.05)$ : T lymphocytes vs. NK cells, plasma cells, and monocytes/macrophages; T lymphocytes vs. NK cells, plasma cells, and monocytes/ macrophages; NK cells vs. plasma cells.

\section{MMP Expression in Inflammatory Cells}

within $A A A s$

Strong MMP-1-, -3- and -9-positive staining was detected in all inflammatory cells (fig. $5 \mathrm{a}, \mathrm{c}, \mathrm{f}$, table 1 ). In contrast, none of the inflammatory cells expressed MMP2 (fig. 5b). MMP-7 and - 8 expression was irregular and mainly associated with $\mathrm{T}$ lymphocytes and plasma cells (fig. 5d, e). MMP-13 expression was predominantly associated with plasma cells (fig. 5h), MMP-12 was negative in all inflammatory cells with the exception of monocytes/macrophages (fig. $5 \mathrm{~h}$ ).

\section{MMP Expression in Macrophages}

Macrophages were positive for all MMPs tested (table 1). However, differences in their expression were detected. Strong expression was observed for MMP-1, -2, -3 and -9 in all macrophages within AAAs. In contrast, staining for MMP-7, -8 and -12 was markedly weaker. Predominantly cholesterol-loaded macrophage-derived foam cells recognized by their granular appearance were positive for MMP-7, -8 and -12. MMP-13 demonstrated the weakest expression among all MMPs tested.

\section{Proteolytic MMP-Mediated Aneurysmal Wall Degradation}

To verify the hypothesis that enhanced accumulation of inflammatory cells and neovascularization are associated with the degradation of AAA structure proteins, infiltrates, neovessels and macrophages were correlated with collagen and elastin content (table 3). A significant positive correlation was found between the amount of inflammatory cells and neovessels $(r=+0.806, p<0.001)$. Furthermore, a significant negative relationship was observed between infiltrates or neovessels and collagen or elastin content $(\mathrm{r}=-0.650, \mathrm{p}=0.012$ and $\mathrm{r}=-0.636, \mathrm{p}=$ $0.015 ; \mathrm{r}=-0.542, \mathrm{p}=0.042$ and $\mathrm{r}=-0.488, \mathrm{p}=0.049$, respectively).
Table 3. Correlation between MMPs, TIMPs and other clinical parameters

\begin{tabular}{llllll}
\hline & Infiltrates & $\begin{array}{l}\text { Macro- } \\
\text { phages }\end{array}$ & Collagen & Elastin & $\begin{array}{l}\text { Neovascu- } \\
\text { larization }\end{array}$ \\
\hline Infiltrates & - & & & & \\
Macrophages & n.c. & - & & & \\
Collagen & $-0.636^{*}$ & n.c. & - & & \\
Elastin & $-0.650^{*}$ & n.c. & $+0.690^{* *}$ & - & - \\
Neovascularization & $+0.806^{* * *}$ & n.c. & $-0.542^{*}$ & $-0.488^{*}$ & - \\
\hline
\end{tabular}

Significant differences between individual biomarkers: ${ }^{*} \mathrm{p}<0.05,{ }^{* *} \mathrm{p}<0.01,{ }^{* * *} \mathrm{p}<$ 0.001 ; n.c. $=$ no correlation . 

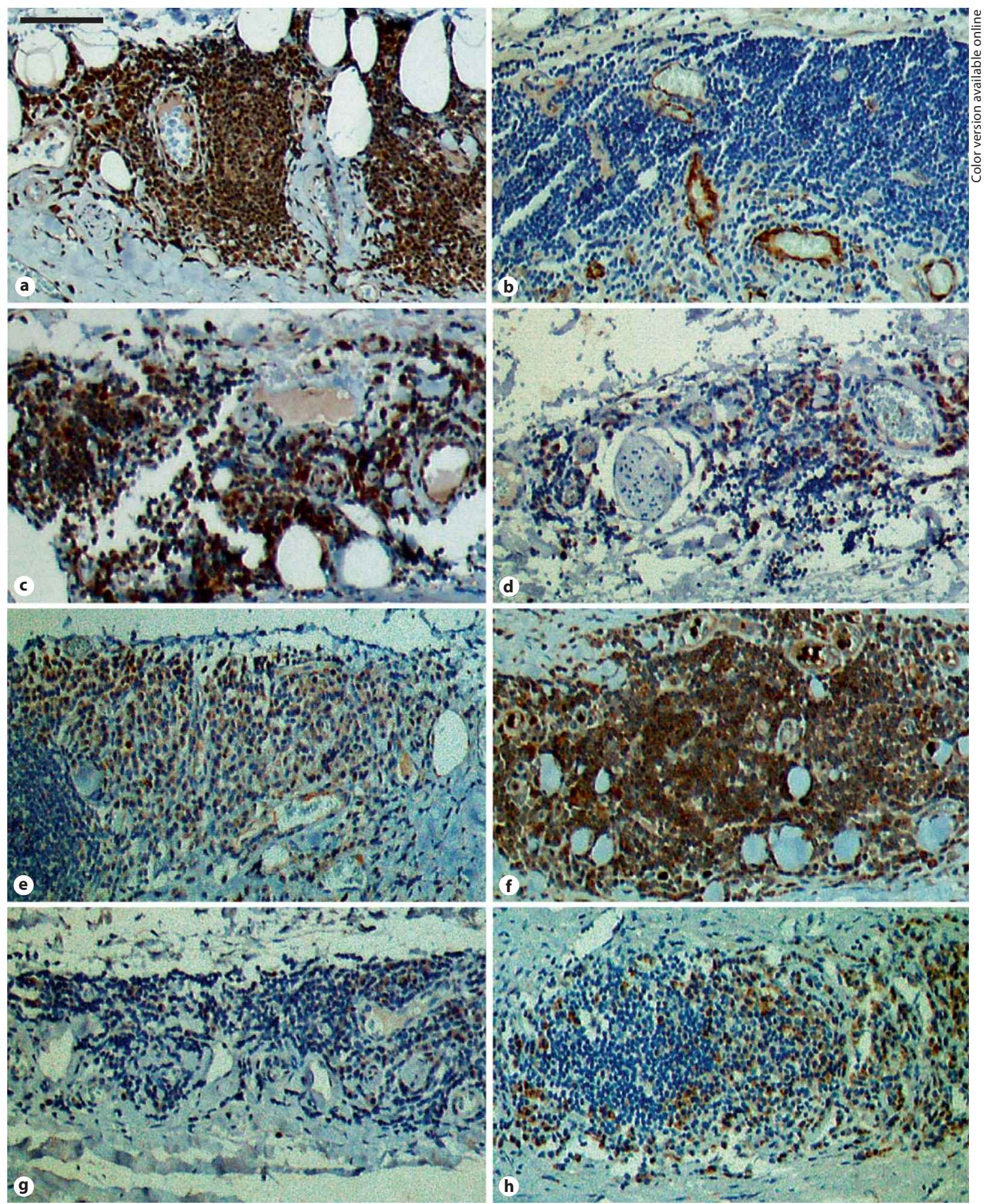

Fig. 5. Immunohistochemical staining of MMP-1 (a), MMP-2 (b), MMP-3 (c), MMP-7 (d), MMP-8 (e), MMP-9 (f), MMP$12(\mathbf{g})$ and MMP-13 (h) within inflammatory infiltrates of human AAA samples. The bar in a corresponds to $100 \mu \mathrm{m}$ and also applies to $\mathbf{b}-\mathbf{h}$. 


\section{Discussion}

MMPs play a decisive role in the pathogenesis of aortic aneurysm formation and rupture [5, 6, 9, 25]. Most research has so far focused on the expression of these proteinases by resident luminal ECs, medial SMCs and invaded macrophages $[4-7,10-16]$. The relevance of inflammatory cells and neovessels in the AAA wall is still unclear.

Our results demonstrated MMP-1 and -9 expression, and partially MMP-3 in both luminal ECs and medial SMCs of the aortic vessel wall. In contrast, MMP-2 was expressed only by medial SMCs. Thus, in view of substrate specificity, ECs and SMCs within the aortic vessel wall may contribute to the degradation of major extracellular matrix components such as collagen or elastin (MMP-1, -2 and -9), fibronectin and proteoglycans (MMP-3) [25, 27-30]. Furthermore, MMP-3 is able to activate other MMPs [28, 31].

In accordance with previously published results, macrophages were positive for all MMPs tested [25]. However, differences in their expression were observed. Strong expression of MMP-1, -2, -3 and -9 was found in all macrophages within AAAs. The immunohistochemical staining of MMP-7, $-8,-12$ and -13 was markedly weaker and mainly associated with atherosclerotic macrophage-derived foam cells. These discrepancies might be explained by deregulation of gene expression and activation of MMPs following uptake of oxidized lipoproteins or extracellular lipids [32, 33].

Accumulation of inflammatory cells is one of the main characteristics of the diseased AAA wall $[9,20]$. However, there appears to be disagreement concerning the composition of inflammatory infiltrates [21, 23, 24]. In our study, B cells were found as the predominant component of inflammatory infiltrates followed by $\mathrm{T}$ lymphocytes and plasma cells. Other cells such as NK cells and monocytes/macrophages represented less than $5 \%$ of the total inflammatory cells. However, the individual and interindividual composition of the infiltrates varied markedly (see ranges in table 2). The presence of these cells demonstrates an autoimmune reaction and may markedly contribute to the pathogenesis of AAAs [22]. Thus, damage to the aortic wall is also the consequence of an immune response and activation of inflammatory cells. Upon activation, $\mathrm{T}$ and $\mathrm{B}$ lymphocytes express a variety of cytokines and promote MMP expression as shown in our study.

Interestingly, aneurysmal infiltrates expressed all MMPs tested with the exception of MMP-2. Strong posi- tive staining for MMP-1, -3 and -9 was detected in all inflammatory cells. In contrast, the staining intensity of MMP-7 and -8 markedly varied between negative and positive expression even in the same specimen. The reason for this heterogeneity in detail is unknown, but analysis of inflammatory cells in correlation with MMP expression revealed that MMP-7 and -8 activities were predominantly associated with $\mathrm{T}$ lymphocytes and plasma cells. Consequently, inflammatory cells within the AAA can considerably contribute to the proteolytic destabilization of the vessel wall.

Medial and intimal neovascularization is a known characteristic of advanced AAAs [6, 8, 17-19]. However, the patterns of MMP expression in these neovessels are still unknown. In our study, all histological samples demonstrated increased neovascularization in the medial and intimal layer compared to the control group. In the media of healthy aortic tissue, no neovessels were detected. Only at the border between media and adventitia were small vessels from the vasa vasorum observed. Furthermore, in accordance with previous reports, most of the neovessels were immature $[6,8]$. These microvessels are generally associated with increased vessel wall permeability, enhanced recruitment of inflammatory cells and presumably with increased MMP expression [6]. Our results showed higher MMP activity in ECs of immature neovessels compared to aortic luminal ECs and medial SMCs. Immature neovessels expressed all MMPs tested except for MMP-13 with the strongest expression for MMP-1, -3 and -9. Interestingly, MMP-2, $-7,-8$ and -12 activities varied markedly between individual neovessels. This variability may depend on their current status. Invading neovessels might express more MMPs than latent ones. This hypothesis was confirmed by the observation that ECs and SMCs of mature neovessels and luminal ECs and medial SMCs showed similar MMP expression. Thus, immature neovessels may also contribute to proteolytic degradation of extracellular matrix. Furthermore, we have found a significant correlation between the content of inflammatory cells and neovessels. In addition, significant negative correlations were observed between the collagen elastin content of the aneurysmal vessel wall and the density of inflammatory infiltrates or neovessels. This finding is concordant with the hypothesis that neovascularization is followed by accumulation of inflammatory cells and consequent expression of various MMPs. These processes may markedly contribute to the rupture of AAAs. 


\section{Limitations}

Despite the extended optimization of all Abs used in our study, the statements regarding different expression patterns of MMPs also depend on the quality of the individual histological specimens and the sensitivity of immunohistochemistry. MMP expression in plasma cells might be partly unspecific due to the high amount of immunoglobulins within these cells. However, control staining was always negative and the staining of the plasma cells was well defined. Therefore, we believe that plasma cells are also able to express MMPs. Similar to other inflammatory cells, plasma cells migrate to the focus of inflammation, which presupposes degradation of surrounding tissue for instance by MMPs.

In summary, our study provided a detailed analysis of all relevant members of soluble-MMP subgroups in all cells within AAAs. Our results show that MMP-1, -3 and -9 are the most commonly expressed MMPs within the
AAA wall. Furthermore, in addition to the well-known MMP activity in luminal ECs, medial SMCs and macrophages, both inflammatory infiltrates and neovessels are relevant sources of MMPs as well and may therefore also contribute to the degradation of the extracellular matrix and rupture of AAAs. This finding is important because invading neovessels lead to accumulation of inflammatory cells. These cells not only stimulate MMP expression by cytokine production, but are able to express a variety of MMPs autonomously. All these processes may lead to accelerated degradation of AAAs and favour their rupture.

\section{Acknowledgments}

This work was in part funded by the 'Doktor Robert PflegerStiftung'. Furthermore, the authors particularly thank Renate Hegenloh for her excellent technical support in the histological and immunohistochemical staining.

\section{References}

1 Choke E, Cockerill G, Wilson WR, Sayed S, Dawson J, Loftus I, Thompson MM: A review of biological factors implicated in abdominal aortic aneurysm rupture. Eur J Vasc Endovasc Surg 2005;30:227-244.

-2 Kniemeyer HW, Kessler T, Reber PU, Ris HB, Hakki H, Widmer MK: Treatment of ruptured abdominal aortic aneurysm, a permanent challenge or a waste of resources? Prediction of outcome using a multi-organdysfunction score. Eur J Vasc Endovasc Surg 2000;19:190-196.

-3 Shimizu K, Mitchell RN, Libby P: Inflammation and cellular immune responses in abdominal aortic aneurysms. Arterioscler Thromb Vasc Biol 2006;26:987-994.

4 Galis ZS, Sukhova GK, Lark MW, Libby P: Increased expression of matrix metalloproteinases and matrix degrading activity in vulnerable regions of human atherosclerotic plaques. J Clin Invest 1994;94:2493-2503.

5 Keeling WB, Armstrong PA, Stone PA, Bandyk DF, Shames ML: An overview of matrix metalloproteinases in the pathogenesis and treatment of abdominal aortic aneurysms. Vasc Endovascular Surg 2005;39:457-464.

6 Thompson MM, Jones L, Nasim A, Sayers RD, Bell PRF: Angiogenesis in abdominal aortic aneurysms. Eur J Vasc Endovasc Surg 1996;11:464-469.

7 Thompson MM: Controlling the expansion of abdominal aortic aneurysms. Br J Surg 2003;90:897-898.
-

Choke E, Thompson MM, Dawson J, Wilson WR, Sayed S, Loftus IM, Cockerill GW: Abdominal aortic aneurysm rupture is associated with increased medial neovascularization and overexpression of proangiogenic cytokines. Arterioscler Thromb Vasc Biol 2006;26:2077-2082

-9 Sakalihasan N, Limet R, Defawe OD: Abdominal aortic aneurysm. Lancet 2005;365: 1577-1589.

$\checkmark 10$ McMillan WD, Tamarina NA, Cipollone M, Johnson DA, Parker MA, Pearce WH: Size matters: the relationship between MMP-9 expression and aortic diameter. Circulation 1997;96:2228-2232.

- 11 Papalambros E, Sigala F, Georgopoulos S, Menekakos C, Giatromanolaki A, Bastounis E, Sivridis E: Immunohistochemical expression of metalloproteinases MMP-2 and MMP-9 in abdominal aortic aneurysms: correlation with symptoms and aortic diameter. Int J Mol Med 2003;12:965-968.

12 Petersen E, Gineitis A, Wagberg F, Angquist KA: Activity of matrix metalloproteinase-2 and -9 in abdominal aortic aneurysms. Relation to size and rupture. Eur J Vasc Endovasc Surg 2000;20:457-461.

13 Petersen E, Wagberg F, Angquist KA: Proteolysis of the abdominal aortic aneurysm wall and the association with rupture. Eur J Vasc Endovasc Surg 2002;23:153-157.

14 Wilson WR, Schwalbe EC, Jones JL, Bell PR Thompson MM: Matrix metalloproteinase 8 in the pathogenesis of abdominal aortic aneurysm. Br J Surg 2005;92:828-833.
15 Wilson WR, Anderton M, Schwalbe EC, Jones JL, Furness PN, Bell PR, Thompson MM: MMP- 8 and -9 are increased at the site of abdominal aortic aneurysm rupture. Circulation 2006;113:438-445.

-16 Kazi M, Zhu C, Roy J, Paulsson-Berne G, Hamsten A, Swedenborg J, Hedin U, Eriksson P: Difference in matrix-degrading protease expression and activity between thrombus-free and thrombus-covered wall of abdominal aortic aneurysm. Arterioscler Thromb Vasc Biol 2005;25:1341-1346.

17 Kobayashi M, Matsubara J, Matsushita M, Nishikimi N, Sakurai T, Nimura Y: Expression of angiogenesis and angiogenic factors in human aortic vascular disease. J Surg Res 2002;106:239-245.

18 Paik DC, Fu C, Bhattacharya J, Tilson MD: Ongoing angiogenesis in blood vessels of the abdominal aortic aneurysm. Exp Mol Med 2004;36:524-533

19 Vorp DA, Lee PC, Wang DH, Makaroun MS, Nemoto EM, Ogawa S, Webster MW: Association of intraluminal thrombus in abdominal aortic aneurysm with local hypoxia and wall weakening. J Vasc Surg 2001;34:291299.

20 Lindholt JS, Shi GP: Chronic inflammation, immune response, and infection in abdominal aortic aneurysms. Eur J Vasc Endovasc Surg 2006;31:453-463. 
-21 Henderson EL, Geng YJ, Sukhova GK, Whittemore AD, Knox J, Libby P: Death of smooth muscle cells and expression of mediators of apoptosis by $\mathrm{T}$ lymphocytes in human abdominal aortic aneurysms. Circulation 1999; 99:96-104.

-22 Pearce WH, Koch AE: Cellular components and features of immune response in abdominal aortic aneurysms. Ann NY Acad Sci 1996;800:175-185.

23 Bobryshev YV, Lord RS, Parsson H: Immunophenotypic analysis of the aortic aneurysm wall suggests that vascular dendritic cells are involved in immune responses. Cardiovasc Surg 1998;6:240-249.

-24 Forester ND, Cruickshank SM, Scott DJ, Carding SR: Functional characterization of $\mathrm{T}$ cells in abdominal aortic aneurysms. Immunology 2005;115:262-270.
25 Newby AC: Dual role of matrix metalloproteinases (matrixins) in intimal thickening and atherosclerotic plaque rupture. Physiol Rev 2005;85:1-31.

26 World Medical Association Declaration of Helsinki. Cardiovasc Res 1997;35:2-3.

27 Schönbeck U, Mach F, Sukhova GK, Atkinson E, Levesque E, Herman M, Graber P, Basset P, Libby P: Expression of stromelysin3 in atherosclerotic lesions: regulation via CD40-CD40 ligand signaling in vitro and in vivo. J Exp Med 1999;189:843-853.

28 Visse R, Nagase H: Matrix metalloproteinases and tissue inhibitors of metalloproteinases: structure, function, and biochemistry. Circ Res 2003;92:827-839.

29 Lemaitre V, D’Armiento J: Matrix metalloproteinases in development and disease. Birth Defects Res C Embryo Today 2006;78: $1-10$.
30 Newby AC: Matrix metalloproteinases regulate migration, proliferation, and death of vascular smooth muscle cells by degrading matrix and non-matrix substrates. Cardiovasc Res 2006;69:614-624.

31 Dollery CM, Libby P: Atherosclerosis and proteinase activation. Cardiovasc Res 2006; 69:625-635.

>32 Galis ZS, Sukhova GK, Kranzhöfer R, Clark S, Libby P: Macrophage foam cells from experimental atheroma constitutively produce matrix-degrading proteinases. Proc Natl Acad Sci USA 1995;92:402-406.

33 Vogel CF, Sciullo E, Matsumura F: Activation of inflammatory mediators and potential role of ah-receptor ligands in foam cell formation. Cardiovasc Toxicol 2004;4:363373. 